\title{
Intersections
}

Canadian Journal of Music

Revue canadienne de musique

\section{Michelle Phillipov. 2012. Death Metal and Music Criticism : Analysis at the Limits. Plymouth (Royaume-Uni) : Lexington Books, 158 p., bilbliogr., discogr., index. 978-07391-6461-7}

\section{Méi-Ra St-Laurent}

Volume 35, numéro 1, 2015

URI : https://id.erudit.org/iderudit/1038955ar

DOI : https://doi.org/10.7202/1038955ar

Aller au sommaire du numéro

Éditeur(s)

Canadian University Music Society / Société de musique des universités canadiennes

ISSN

1911-0146 (imprimé)

1918-512X (numérique)

Découvrir la revue

Citer ce compte rendu

St-Laurent, M.-R. (2015). Compte rendu de [Michelle Phillipov. 2012. Death Metal and Music Criticism : Analysis at the Limits. Plymouth (Royaume-Uni) :

Lexington Books, 158 p., bibliogr., discogr., index. 978-07391-6461-7].

Intersections, 35(1), 185-191. https://doi.org/10.7202/1038955ar

Copyright @ Canadian University Music Society / Société de musique des universités canadiennes, 2016
Ce document est protégé par la loi sur le droit d'auteur. L'utilisation des services d'Érudit (y compris la reproduction) est assujettie à sa politique d'utilisation que vous pouvez consulter en ligne.

https://apropos.erudit.org/fr/usagers/politique-dutilisation/ 
development, and this publication is both a testament to his investment in that community as well as a generous invitation for us to join them.

ERIC SMIGEL

\section{BIOGRAPHY}

Eric Smigel is Associate Professor of Music and Coordinator of the Musicology Program at San Diego State University, where he teaches courses in music history, music research, and a general education course in Psychedelic Rock of the 196os. He has published articles, reviews, and liner notes on various topics of American experimental music, including studies of John Cage, David Tudor, James Tenney, Joseph Byrd, and Lois V Vierk. Eric is currently under contract with the University of Illinois Press to write a book-length biography of Tenney, which will highlight the composer's relationship with independent filmmaker Stan Brakhage and visual artist Carolee Schneemann. He received the M.A. degree in Music History and Ph.D. in Historical Musicology from the University of Southern California.

Michelle Phillipov. 2012. Death Metal and Music Criticism: Analysis at the Limits. Plymouth (Royaume-Uni) : Lexington Books, 158 p., bibliogr., discogr., index. 978-07391-6461-7.

\section{Dans son ouvrage Death Metal and Music Criti-} cism: Analysis at the Limits, Michelle Phillipov ${ }^{1}$ porte un regard critique sur la manière dont les musiques populaires sont généralement envisagées en études culturelles en prenant pour exemple le cas du death metal ${ }^{2}$. Dans la première des deux parties de l'ouvrage, Phillipov remet en question la lecture politique des principaux courants de musique populaire (punk, hip-hop, electro dance music [EDM], etc.), qui demeure généralement le fondement de toute analyse. En effet, elle explique qu'en se limitant à une telle approche (même si elle demeure pertinente), plusieurs chercheurs mettent de côté un des éléments les plus fondamentaux de la

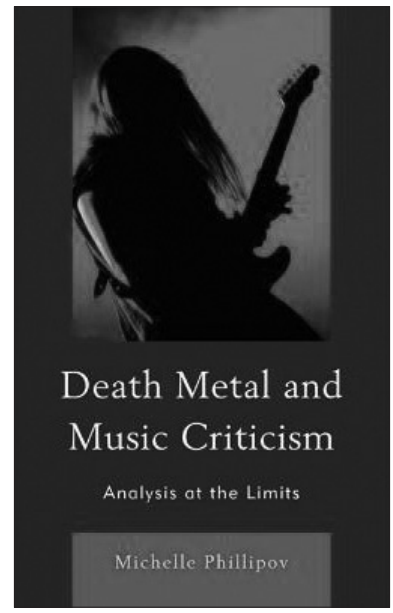

1 Phillipov est chargée de cours à l'Université de Tasmanie (Australie) en journalisme, média et communication.

2 Le death metal est un style musical appartenant au metal extrême, s'étant développé vers la fin des années 1980 (particulièrement aux États-Unis et en Suède), et qui se caractérise par un rythme très rapide à la batterie (blast beat), une sonorité lourde et claire à la guitare et une voix grognée (voix growl) très grave. La complexité des progressions harmoniques, mélodiques et rythmiques définit ce style et exige une grande virtuosité de la part des musiciens. Les thèmes abordés par les premiers groupes de death metal (p.ex. décapitation, cannibalisme, éviscération) sont d'une violence crue et explicite, et visent à transgresser les normes musicales et textuelles non seulement de la musique metal, mais de l'ensemble des musiques populaires. 
musique populaire: la dimension du plaisir ressenti par les amateurs. Ainsi, l'auteure s'appuie sur les groupes fondateurs du death metal (Carcass, Cannibal Corpse, Napalm Death, pour ne nommer que les plus connus) pour démontrer que le discours politique est souvent absent ou situé au second plan, justifiant alors d'aller au-delà de cette approche pour parvenir à une meilleure compréhension de la musique metal. Comme alternative, dans la seconde partie de son ouvrage, Phillipov examine les différentes conventions musicales et textuelles rattachées aux groupes de death metal à l'étude, renforçant l'hypothèse que le death metal soit avant tout une source de plaisir sonique sans qu'il n'y ait nécessairement de structure analytique ou de récits cohérents sous-jacents.

D’emblée, Phillipov ouvre le débat avec une question d'une grande pertinence: pourquoi l'aspect politique est-il si important dans l'analyse de la musique populaire? Cela s'explique principalement par le fait que les études culturelles sont parmi les premières branches des sciences humaines à s'être intéressées à la musique populaire et qu'historiquement, le but de ce champ d'études a été d'affirmer l'aspect subversif de la musique populaire en prenant comme point d'ancrage la résistance de la classe ouvrière envers la culture hégémonique. Plus spécifiquement, la majorité des approches développées dès la formation du champ des études culturelles (p. ex. le concept de résistance à travers le rituel (Hall et Jefferson 1976) ou de la notion de sous-culture (Hebdige 1979) se sont basées sur la politique, ce qui, en plus de donner le ton aux recherches ultérieures, a limité les autres approches analytiques possibles.

Ainsi, les universitaires envisagent généralement le punk, le hip hop et l'EDM comme des représentations symboliques de résistance communautaire à l'ordre social hégémonique. Le punk, genre apprécié pour sa sonorité agressive, est souvent associé à la classe ouvrière pour ses thèmes revendicateurs opposés aux idéologies de l'extrême droite. Toutefois, Phillipov rappelle que les premiers groupes punks influents (p. ex. Sex Pistols) proviennent de la classe moyenne et non de la classe ouvrière et qu'il existe une filiation solide entre le néonazisme et le punk dès les années 1970. En ce qui a trait au hip hop, les études passées véhiculent l'idée que le rap étant "parlé», il permet de mieux transmettre les idées politiques des chansons et renforce l'interaction et la fierté des communautés afro-américaines. Toutefois, cette réflexion devient caduque lorsqu'on s'intéresse à la majorité blanche d'amateurs de rap, n'étant ni concernée ni interpelée par le contenu politique des paroles (Yousman 2003). Dans le cas de l'EDM, le plaisir désengagé ressenti lors des raves permet certes de questionner les barrières sociales radicales, puisque les personnes habituellement marginalisées sont ici acceptées. Toutefois, comment expliquer en termes de résistance politique le succès lucratif et non communautaire des soirées raves? Ainsi, les pratiques culturelles ne semblent pas toujours se rattacher aux positions politiques interprétées dans un cadre universitaire préconçu. En outre, les universitaires ont tendance à considérer ces conventions musicales et textuelles comme étant fondamentales, évacuant alors toute la dimension du plaisir éprouvé par les amateurs. Toutefois, l'auteure démontre que l'analyse du heavy metal permet de renverser cette tendance. 
Les études universitaires portant sur le heavy metal débutent tardivement, soit au début des années 1990, avec les ouvrages de Deena Weinstein (1991) et Robert Walser (1992). Ces deux auteurs demeurent particulièrement importants, puisqu'ils portent un regard neuf sur le heavy metal, en tentant d'en appréhender ses mécanismes de manière objective. En effet, les autres universitaires s'y intéressant durant cette période (Cashmore 1987; Gaines 1998) le considèrent non seulement d'un point de vue extérieur, mais critiquent son manque d'engagement sur le plan politique. Or, le heavy metal est compris comme étant axé sur la colère, sur la fuite de l'engagement, et le plaisir qu'il semble procurer est interprété comme étant nihiliste et destructeur. Ainsi, lorsqu'on le compare au hip-hop ou au punk, le metal est considéré comme réactionnaire, improductif et difficile d'accès musicalement. Toutefois, Phillipov rappelle que l'objectif du metal n'a jamais été de changer la société ou ses structures politiques (même si elles sont souvent désapprouvées), mais d'y échapper momentanément à travers une forme d'écoute alternative procurant du plaisir aux auditeurs en déchiffrant les codes.

Phillipov explique donc que le death metal n'aborde pas l'aspect politique avant tout parce qu'une grande majorité des musiciens et des amateurs refusent d'interroger les implications politiques de la musique qu'ils produisent et consomment. Afin de mieux décrire ce comportement, Phillipov se base sur le concept développé par Keith Kahn-Harris, soit l'attitude «réflexive

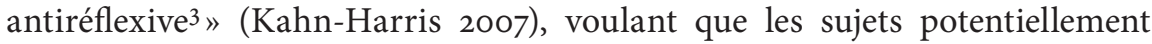
problématiques abordés dans les chansons de metal extrême (p. ex. propos racistes, violence gratuite) soient consciemment ignorés par les musiciens et les amateurs au profit du plaisir désengagé pour la musique. Pour le dire plus simplement, l'attitude réflexive antiréflexive réoriente complètement l'écoute musicale.

Ainsi, Phillipov s'attache à démontrer que les structures musicales narratives traditionnelles sont abandonnées au profit d'autres conventions musicales à l'intérieur desquelles le texte n'est pas considéré comme étant un élément cohérent. Pour justifier la faible proportion d'éléments narratifs du death metal, l'auteure s'intéresse d'abord à la voix grognée 4 caractérisant le death metal. Elle prend appui sur le concept du "grain de la voix» de Barthes (1981): "In Barthes's work, "grain" is used as a designation for the timbral qualities of vocal sound in which the materiality of the voice [...] interrupts or exceeds meaning: the moment in which meaning and signification are overwhelmed by music's jouissance» (p. 79) . En outre, dans le death metal, la voix est tellement déformée et dénaturalisée, qu’elle excède sa fonction communicative pour devenir uniquement expressive. Les paroles sont donc souvent considérées comme un élément secondaire, qui ne doit pas nuire aux éléments instrumentaux ou au plaisir du son vocal en lui-même. En outre, même lorsque les

3 Ma traduction («Reflexive anti-reflexive attitude»).

4 La voix grognée se caractérise par un grognement très grave semblable à celui d'un animal et est habituellement employée pour exprimer la colère. Dans la musique metal, ce timbre personnifie généralement des créatures maléfiques et induit une impression tant de puissance que d'oppression (Brizard 2006, 124; 126). 
paroles sont structurées de manière cohérente et que la voix semble avoir une fonction communicative, les paroles demeurent difficilement compréhensibles, rendant toute interprétation politique incertaine. Phillipov effectue d'ailleurs un rapprochement entre l'expérience esthétique du death metal, qui demeure similaire à celle de l'amateur de films d'horreur de type "gore». En effet, ce type de cinéma ne propose qu'une trame narrative superficielle au profit de mises en scène sanglantes et explicites. À cet égard, Phillipov considère que la récurrence de certains sujets permet d'avancer que ce sont les thèmes abordés, davantage que les paroles, qui demeurent importants (p. 101-104).

Dans le cas du death metal, Phillipov note que les thèmes présentés dans les chansons de certains groupes n'offrent souvent pas de cadre narratif précis permettant de rendre compte de l'action. Prenant pour exemple la chanson «Shredded Humans» de Cannibal Corpse (1990), où un tueur massacre une famille, Phillipov démontre que les informations contextuelles permettant à l'auditeur d'établir une trame narrative claire, comme l'identification du tueur, des victimes, des lieux, demeurent insuffisantes. Ainsi, le fait que le death metal soit très graphique tout en étant décontextualisé (comme le cinéma gore) permet de dire que ce style n'est pas lié à une fin narrative spécifique. Au contraire, il doit plutôt être appréhendé comme un spectacle sonore, lyrique et graphique provoquant une expérience de sensations extrêmes chez les amateurs, qui s'apparente à la catharsis, tout en étant complètement fictif et coupé de toutes les préoccupations quotidiennes. En effet, les images d'horreurs véhiculées dans le death metal doivent donc être interprétées davantage en relation avec l'histoire du genre et ses conventions, et non comme une manière d'encourager la reproduction de comportements violents. Pour Phillipov, c'est cette réorientation de l'écoute qui permet aux amateurs de se désengager à l'égard du discours violent véhiculé par le death metal.

Enfin, le death metal présente une esthétique sonique extrême où l'auditeur est invité à apprécier la complexité et l'agressivité de la sonorité death metal, habituellement considérée comme du bruit par les néophytes. Plus spécifiquement, les riffs sont employés comme des éléments structurels, où leur enchâ̂nement tantôt rapide, tantôt lent constitue la charpente de la chanson. Celle-ci sera elle-même renforcée par un rythme de batterie difficilement prévisible et extrêmement rapide, le tout joint à un texte incompréhensible, mais dont la voix demeure appréciée pour son agressivité. L'imprédictibilité du death metal n'offre donc aux auditeurs aucune stabilité ou récurrence (p. ex. la forme couplet-refrain propre à la musique populaire) comme point d'attache. Ainsi, pour les amateurs, le plaisir procuré par le death metal réside aussi dans sa complexité et son inaccessibilité.

En somme, l'auteure avance que les conventions du death metal (l'attitude réflexive antiréflexive, l'agressivité musicale et thématique, les capacités techniques), plutôt que seulement l'opposition à une hiérarchie sociale, peuvent être appréhendées comme des moyens pour créer une expérience d'écoute différente, ne pouvant être évaluées en terme de «progressiste» ou « réactionnaire». 
Ainsi, cet ouvrage soulève plusieurs questionnements pertinents, qui permettront sans doute de remettre en question certains fondements idéologiques propres à l'analyse de la musique populaire. Toutefois, quelques points liés à l'analyse narrative du death metal demeurent ambigus. En effet, l'ouvrage présente une lacune au niveau méthodologique, car l'analyse du récit ne semble se baser sur aucun des modèles éprouvés dans le domaine de la musique populaire5. On pourrait donc s'interroger sur la fiabilité des résultats des analyses effectuées, qui n'auraient peut-être pas mené aux mêmes conclusions si l'auteure avait employé des outils ayant fait leur preuve. Par exemple, le premier couplet de la chanson «Shredded Humans» présenté ci-dessous comporte tous les éléments propres au récit: on retrouve un cadre temporel (une mise en contexte de l'endroit et du moment où se déroule l'action), un narrateur (extérieur à l'action, celui-ci met en scène chacun des personnages) et un discours (présenté ici sous la forme d'une narration des événements à venir).

Early hours, open road, family of five-on their way home

Having enjoyed a day in the sun, their encounter with gore has just begun

A homicidal fool not knowing left from right, now has the family in his sight

Trying to perceive if he's blind or insane,

he steers his car into the other lane [...]

Malgré le fait qu'une trame narrative soit présente, ce récit est volontairement obscurci par l'utilisation du death metal, comme le signale Phillipov ${ }^{6}$. Toutefois, on remarque que l'analyse du récit par l'auteure demeure liée à la seule réception des amateurs et omet toute la portion du processus créatif des musiciens. En effet, il aurait été intéressant de savoir si les musiciens des groupes sélectionnés considèrent leur musique comme porteuse d'un discours ou d'un récit précis, puisque dans le cas de «Shredded Humans», il y en a bien un. Cependant, il est vrai que les groupes sélectionnés par l'auteure appartiennent à une catégorie spécifique de death metal, où les termes utilisés dans les chansons sont souvent sélectionnés pour leur «sonorité phonétique agressive». Le sens des phrases est alors volontairement obscurci pour accroître la dimension malsaine du propos, la trame narrative passant forcément au second plan.

Enfin, il est à noter que cette volonté de rester en marge d'un discours politique ne fait pas consensus au sein de la scène de death metal. Ainsi, plusieurs groupes (p. ex. Sepultura, Gorguts, Lamb of God) proposent une réflexion politique approfondie (et une trame narrative élaborée dans certains cas) ce qui pourrait laisser entendre que les paroles jouent un rôle prépondérant pour ces groupes. Malgré ces quelques éléments, il n’en demeure pas moins que cet

5 Les principaux modèles analytiques offerts sont ceux de Gianni Sibilla (2003), de Serge Lacasse (2006), qui sont basés sur la théorie de la narratologie de Gérard Genette, ainsi que celui de Keith Negus (2007), qui reprend la notion de récit de Paul Ricoeur.

6 À mon sens, l'utilisation du death metal pour la transmission d'un récit violent, même si certains passages demeurent difficilement compréhensibles, est nécessaire pour assurer la crédibilité de ce récit. Ainsi, le même propos retransmis, par exemple, en utilisant la musique country \& western ne saurait être aussi efficace. 
ouvrage est tout à fait novateur dans son genre, puisqu'à travers le death metal, un style souvent ignoré, l'auteure porte un regard critique sur la manière dont la musique populaire est généralement envisagée dans le monde académique.

Méi-Ra St-Laurent

\section{RÉFÉRENCES}

Barthes, Rolland. 1981. Le grain de la voix: Entretiens 1962-1980. Paris: Éditions du Seuil.

Brizard, Cyril. 2006. «La fusion de la musique metal et de la musique classique: Nightwish et les voix chantées. Une étude de cas». Copyright Volume! 5, $\mathrm{n}^{\mathrm{o}} 2$ : $115-135$.

Cannibal Corpse. 1990. "Shredded Humans». Eaten Back to Life. Disque compact. Metal Blade.

Cashmore, E. Ellis. 1987. «Shades of Black, Shades of White». Popular Music and Communication, dirigé par James Lull, 245-265. Newbury Park: Sage.

Gaines, Donna. 1998. Teenage Wasteland: Suburbia's Dead End Kids. Chicago: University of Chicago Press.

Kahn-Harris, Keith. 2007. Extreme Metal: Music and Culture on the Edge. Oxford: Berg.

Hall, Stuart et Tony Jefferson. 1976. Resistance Through Rituals: Youth Subcultures in Post-War Britain. Londres: Hutchinson.

Hebdige, Dick. 1979. Subculture: The Meaning of Style. Londres: Methuen.

Lacasse, Serge. 2006. «Stratégies narratives dans "Stan" d'Eminem: le rôle de la voix et de la technologie dans l'articulation du récit phonographique». Protée 34, $\mathrm{n}^{\mathrm{O}}$ 2-3: 11-26.

Negus, Keith. 2007. "Narrative Time and Popular Song». Popular Music and Society 35, $\mathrm{n}^{\mathrm{0}}$ 4: 483-500.

Sibilla, Gianni. 2003. «Fragments of Musical / Narratological Practice: Reading Pop Music as a Narrative, Musicians as Characters». Dans Practicing Popular Music: $12^{\text {th }}$ Biennial Conference IASPM-International Conference Montreal 2003 Proceedings, http://www.iaspm.net/archive/IASPMo3sm .pdf (consulté le 12 octobre 2011).

Walser, Robert. 1993. Running with the Devil: Power, Gender and Madness in Heavy Metal Music. Middletown, Conn.: Wesleyan University Press.

Weinstein, Deena. 1991. Heavy Metal: A Cultural Sociology. New York: Maxwell Macmillan International.

Yousman, Bill. 2003. "Blackophilia and Blackophobia: White Youth, the Consumption if Rap Music, and White Supremacy». Communication Theory $13, n^{\circ} 4: 366-391$.

\section{BIOGRAPHIE}

Méi-Ra St-Laurent est doctorante en musicologie de la musique populaire sous la direction de Serge Lacasse à l'Université Laval et boursière du CRSH. Ses intérêts d'étude 
portent sur l'analyse du récit dans la musique métal, dans lequel elle étudie la manière dont la musique et les paroles forment un véritable récit, souvent à portée transgressive. Dans le cadre de sa thèse de doctorat, elle s'intéresse à la scène de black metal en provenance du Québec, où elle dresse des liens entre le récit musical présent dans les chansons des différents groupes et le discours entretenu par les membres de la scène par rapport à ce récit. 